\title{
Testing SPI imaging of high-energy and extended sources
}

\author{
C. B. Wunderer ${ }^{1}$, P. Connell ${ }^{2}$, J. W. Hammer ${ }^{3}$, V. Schönfelder ${ }^{1}$, and A. W. Strong ${ }^{1}$ \\ 1 Max-Planck-Institute for extraterrestrial Physics, Giessenbachstr. 1, 85748 Garching, Germany \\ 2 University of Birmingham, Edgbaston, Birmingham B15 2TT, UK \\ ${ }^{3}$ Institut für Strahlenphysik, University Stuttgart, Allmandring 3, 70569 Stuttgart, Germany
}

Received 7 July 2003 / Accepted 10 September 2003

\begin{abstract}
INTEGRAL's main instruments employ coded apertures to obtain directional information on the incoming radiation. In order to experimentally better determine the imaging capabilities of the spectrometer SPI, the SPI Imaging Test Setup (SPITS) has been built at MPE. It consists of the SPI coded mask and two SPI-identical Ge detectors on an $X Y$-table which allows us to move them to cover the $19 \mathrm{Ge}$ detector positions. The SPI flight model imaging calibration only covered the energy range up to 2.7 MeV and did not include extended emission. SPITS was used to explore the performance of such a coded aperture system - combined with the SPI image analysis software - for higher-energy point sources and extended sources. We find that a $2.4^{\circ}$ diameter disk emitting $511 \mathrm{keV}$ emission is reconstructed well. For the high signal-to-noise ratios of laboratory measurements, positions of point sources above $4 \mathrm{MeV}$ could be reconstructed to better than $0.1^{\circ}$.
\end{abstract}

Key words. INTEGRAL - spectrometer SPI - instrumentation: imaging - gamma rays

\section{Introduction}

The INTEGRAL spectrometer SPI is designed to measure $20 \mathrm{keV}-8 \mathrm{MeV}$ photons with an energy resolution of $2.3 \mathrm{keV}$ at $1.3 \mathrm{MeV}$ using 19 high-purity Ge detectors. A tungstenalloy coded aperture provides spatial information with an angular resolution of $\sim 2.5^{\circ}$ and a $16^{\circ}$ fully-coded field-of-view (Vedrenne et al. 2003).

Coded apertures, although well established in the X-ray domain (see e.g. Badiali et al. 1985 and references therein), are used by INTEGRAL's instruments for the first time on a space platform up to $10 \mathrm{MeV}$. Studies of the imaging capabilities of SPI were performed using GEANT and other simulation tools (Connell et al. 1998; Strong et al. 1998; Skinner et al. 1997).

The spectrometer has been calibrated in spring 2001 at the accelerator facility of the CEA at Bruyères-le-Châtel, France (Schanne et al. 2002; Attié et al. 2003). These measurements were subject to strict time constraints. Thus some aspects of SPI performance could not be addressed, among them SPI response to extended sources and the imaging performance of the instrument above $3 \mathrm{MeV}$. SPI imaging above a few $\mathrm{MeV}$ is also difficult to test in flight due to low celestial source fluxes at these energies (see Roques et al. 2003).

To complement the SPI calibration data from Bruyèresle-Châtel, the SPI Imaging Test Setup (SPITS) was built at the Max-Planck-Institut für extraterrestrische Physik (MPE). SPITS measurements include imaging data up to $9 \mathrm{MeV}$ taken

Send offprint requests to: C. B. Wunderer, e-mail: wunderer@ssl.berkeley.edu at the Institut für Strahlenphysik (Univ. Stuttgart, Germany) and measurements with an extended $511 \mathrm{keV}$ source.

\section{The SPI Imaging Test Setup}

The SPI Imaging Test Setup (SPITS) consists of two SPI Ge detectors and a SPI-equivalent coded aperture. The Ge detectors are taken from the SPI satellite manufacturing line and mounted in an $\mathrm{Al}$ cryostat. For cost reasons, SPITS has only 2 Ge detectors. The 19-Ge detector camera of SPI is emulated by mounting the Ge detectors on an $X Y$-table. The 19 Ge positions are covered in 11 consecutive measurements. This method necessitates constant source activity over the 11 consecutive measurements. Where this condition is not met, measurement times have to be corrected a posteriori based on monitor data or a known source decay rate. The SPITS coded mask is built on the basis of a SPI mask development model made available by the University of Valencia. All mask materials are identical to those of the SPI mask. The development model was extended to the full SPI mask code and mounted in an Al frame.

The test setup has some restrictions, primarily due to the lack of 17 detectors during any one measurement. With SPITS, the SPI "multiple events (ME)" cannot be used - only photopeak interactions in one Ge detector are used for image analysis. Figures 1 and 2 show pictures of the SPITS mask and Ge detector assemblies. A more detailed description of the setup can be found in Wunderer et al. (2001).

In general, all SPITS measurements have comparatively low (laboratory) background and high count levels, resulting in much higher $\mathrm{S} / \mathrm{N}$-ratios than are expected/seen from 


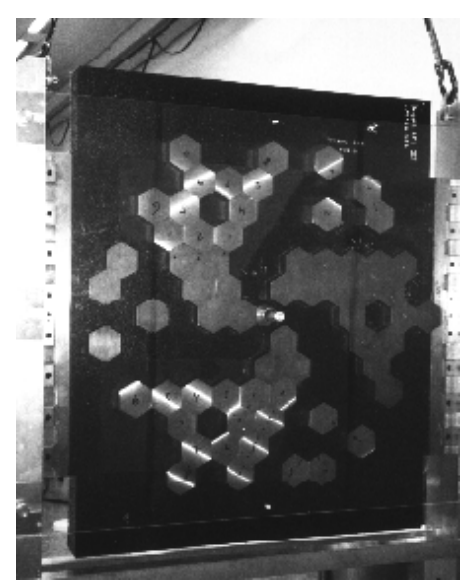

Fig. 1. The SPITS mask with its Al mounting frame.

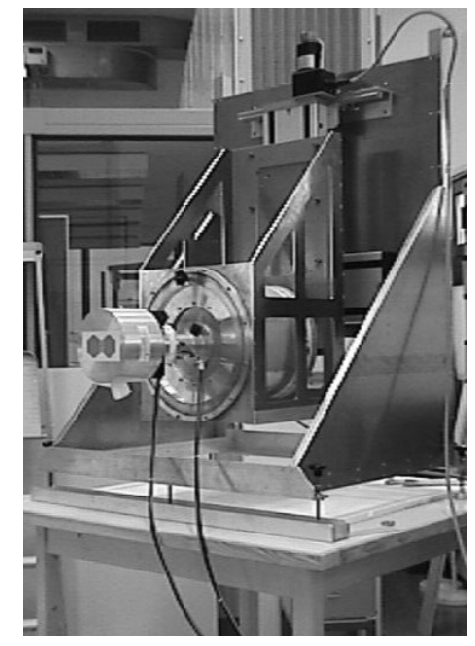

Fig. 2. The SPITS Ge detectors, mounted with their liquid $\mathrm{N}_{2}$ dewar on the $X Y$-table.

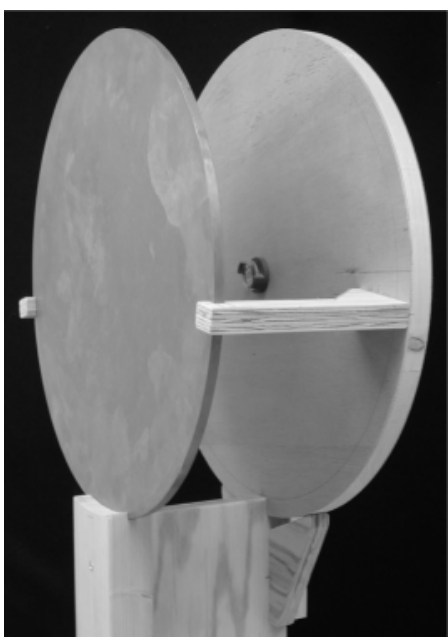

Fig. 3. The $\mathrm{Pb}$ disk used as source of extended emission, shown with ${ }^{88} \mathrm{Y}$ source and mounting device. celestial $\gamma$-ray sources. Reconstructed source significances for the point sources discussed here are $\sim 100 \sigma$ and more.

\section{Imaging algorithms and the SPITS instrument response functions}

Since coded aperture instruments do not produce a "conventional" image of the source, but instead record the source distribution information convolved with the mask function, a deconvolution of the data is needed to recover the spatial source distribution. Combining data from several pointings of the instrument close to the source (region) of interest - a strategy called dithering - usually allows a more unambiguous source reconstruction especially in the case of diffuse emission or crowded fields. For SPI standard analysis, two image reconstruction algorithms have been developed.

Image entropy is a measure for structure in the image. By maximizing this entropy, spiskymax searches for the image with the least deviations from a prior-knowledge distribution (e.g. a flat image) that is consistent with the available data. With increasing number of iterations, spiskymax reconstructions get "spikier", and thus especially for extended emission the stopping criterion is an issue. See Strong et al. (2003) for details on spiskymax.

spiros (SPI Iterative Removal Of Sources) in its basic form is primarily geared towards point sources. A rough sky image is generated by cross-correlation. The strongest point source is located and subtracted in dataspace. This process is repeated until all significant sources are found (Skinner et al. 2003).

In order to reconstruct source images with either algorithm, the instrument response (expected rates in all $19 \mathrm{Ge}$ detectors) to incoming radiation of a given energy has to be known for all possible incident directions. This is stored in Instrument Response Functions (IRFs). For SPI, these IRFs are obtained with MGEANT from a combination of full Monte Carlo simulations and ray-tracing using a complex mass model (Sturner et al. 2003). For SPITS, different IRFs were needed due to a different mass model. The SPI IRF generation tools could not be used since they assumed parallel-beam sources and SPITS measurements were performed with sources at $9 \mathrm{~m}$. SPITS IRFs were generated instead using a ray-tracing method only (CAPTIF, Connell et al. 1995). The resulting SPITS IRFs agree fairly well with measured data once a correction factor is applied to account for the actual Ge photopeak efficiencies. However, since CAPTIF could not accomodate a full SPITS mass model and the presence of 19 detectors had to be assumed, the accuracy of SPITS IRFs is necessarily somewhat less than that of SPI IRFs. Therefore, the performance parameters obtained here with the Test Setup and its IRFs have to be considered lower limits for SPI (and SPI IRF) performance under the same circumstances.

\section{Imaging of point sources above $3 \mathrm{MeV}$}

While SPI is designed to image celestial sources up to $8 \mathrm{MeV}$, it was calibrated on ground with its mask in place only up to $2.7 \mathrm{MeV}$. At higher energies, precedence was given to a uniform energy and efficiency calibration of the Ge detectors over a test of the imaging performance; for this, the mask was removed. Thus, no imaging measurements exist with SPI above 2.7 MeV. To fill this gap, SPITS imaged proton-capture targets $\left({ }^{13} \mathrm{C},{ }^{19} \mathrm{~F}\right.$, and $\left.{ }^{15} \mathrm{~N}\right)$ at the IfS Stuttgart emitting gamma-ray lines up to $9 \mathrm{MeV}$ (Wunderer 2003).

We imaged these "sources" using spiskymax and spiros. In addition to imaging one target, we combined the data from two observations to generate datasets of "two-target observations". This allowed us to test the capability of SPITS and the imaging algorithm used to separate and correctly locate two close point sources of equal intensity. Since absolute target flux calibrations are difficult and there is no independent way to determine the necessary correction factors for the SPITS IRFs, we limit ourselves to the discussion of source positioning for the accelerator targets. 


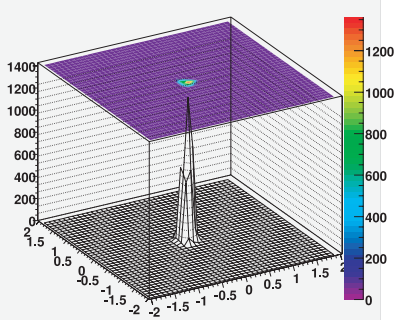

${ }^{13} \mathrm{C}$ target in the $8.1 \mathrm{MeV}$ line at $0.0^{\circ}$ (spiskymax).

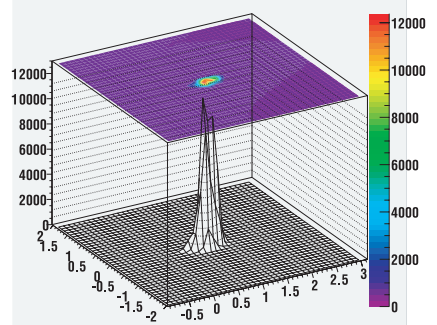

${ }^{19} \mathrm{~F}$ target in the $6.1 \mathrm{MeV}$ line at $1.1^{\circ}$ (spiskymax).

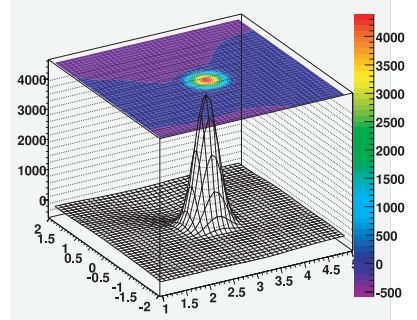

${ }^{13} \mathrm{C}$ target in the $8.1 \mathrm{MeV}$ line at $2.9^{\circ}$ (spiros).

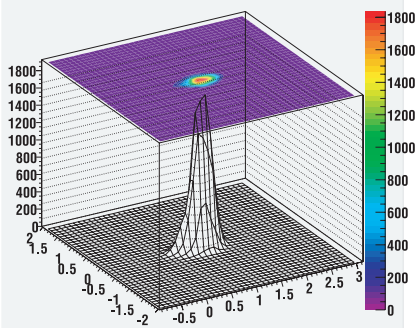

${ }^{19} \mathrm{~F}$ target in the $6.9 \mathrm{MeV}$ line at $1.1^{\circ}$ (spiskymax).

Fig. 4. Reconstructions of accelerator targets with spiskymax and spiros. Photon energies and aspect angles vary.

Figure 4 shows reconstructions of accelerator targets at different photon energies and off-axis angles from SPITS using both spiskymax and spiros algorithms. Single targets were localized well with the spiskymax algorithm in all cases; the location accuracy is $0.1^{\circ}$ or better. Reconstructions with spiros were problematic in many instances; these coincide with measurements taken during periods of unstable accelerator performance. The most likely explanation for this is that the flux corrections applied to the 11 SPITS positions composing one SPITS "observation" are not good enough for these periods of unstable accelerator operations, resulting - in a sense - in different source fluxes seen by different Ge detectors. In this case the IRFs would not reflect the true instrument response (as would happen for an instrument like SPI if the 19 detector efficiencies were poorly known, for example). Thus the results suggest that Maximum Entropy can be more robust than pointsource fitting where the response is not well understood.

Even given these imperfections, two close sources in the field can be separated and localized "correctly" (i.e. to better than $0.2^{\circ}$ ) by the spiskymax algorithm for source separations above $1^{\circ}$. For separations of $0.5^{\circ}$, generally the two sources are not resolved. This is similar to the SPITS findings for lowerenergy point sources. Figure 5 shows spiskymax reconstructions of two-target observations for different separation angles and photon energies.

For normal in-flight conditions, SPI's source location accuracy can be better than $0.1^{\circ}$ for strong sources at low energies (Bouchet et al. 2003), but is more typically $1^{\circ}$ for weaker sources and at higher energies (Attié et al. 2003).

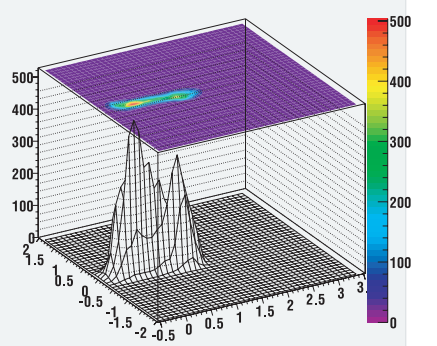

${ }^{13} \mathrm{C}$ targets in the $8.1 \mathrm{MeV}$ line at $0.0^{\circ}$ and $1.0^{\circ}$

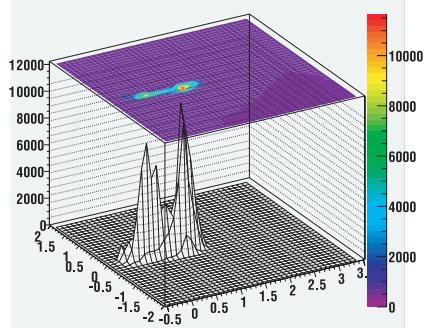

${ }^{19} \mathrm{~F}$ targets in the $6.1 \mathrm{MeV}$ line at $0.0^{\circ}$ and $1.0^{\circ}$

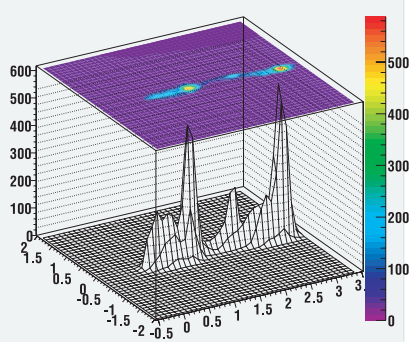

${ }^{13} \mathrm{C}$ targets in the $8.1 \mathrm{MeV}$ line at $1.1^{\circ}$ and $2.9^{\circ}$

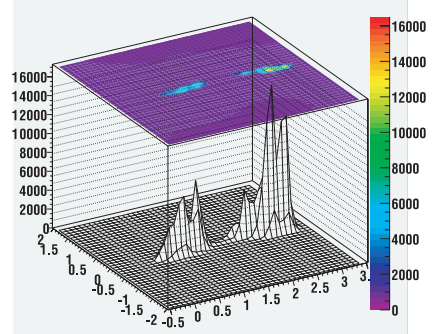

${ }^{19} \mathrm{~F}$ targets in the $6.1 \mathrm{MeV}$ line at $1.1^{\circ}$ and $2.9^{\circ}$

Fig. 5. spiskymax reconstructions of two targets; photon energies and separation angles vary. Source separation is clearer for the ${ }^{19} \mathrm{~F}$ than for the ${ }^{13} \mathrm{C}$ target. This is at least in part due to more photons measured from the ${ }^{19} \mathrm{~F}$ target.

\section{Imaging of extended emission}

To test SPITS' capability to image extended emission, we placed a strong ${ }^{88} \mathrm{Y}$ source behind a lead disk (Fig. 3). In this disk, $1.8 \mathrm{MeV}$ photons from the source interact; some produce electron-positron pairs. The positrons in turn annihilate, and as a result, the whole disk "glows" in $511 \mathrm{keV}$ photons. Of course, $511 \mathrm{keV}$ emission from the center of the disk is stronger than from the disk edge. At $9 \mathrm{~m}$ from the detectors, the disk appears $2.4^{\circ}$ across. An $8 \mathrm{MBq}$ source was used; it produced $55000 \mathrm{phot} / \mathrm{s}$ from the disk or $520511 \mathrm{keV}$ photons incident on one (non-shaded) Ge detector in a one-hour measurement. A more detailed description of the lead disk and the determination of its $511 \mathrm{keV}$ emissivity can be found in Wunderer (2003). Figure 6 shows the $511 \mathrm{keV}$ emission from the disk predicted by an analytical calculation based on first interactions of the $1.8 \mathrm{MeV}$ photons only.

Dithering, as mentioned above, is especially important for observations of extended emission. But even without dithering, SPI with spiskymax can discriminate extended and point source emission given a strong signal and low background. Figures 7 and 8 show reconstructed images of our extended source and a $511 \mathrm{keV}$ point source of equal flux $\left({ }^{22} \mathrm{Na}\right)$.

In order to emulate a 5 -point dither pattern with pointings separated by $2^{\circ}$, all available measurements at five $\mathrm{Pb}$ disk positions were combined. The measurement durations had to be corrected for the ${ }^{88} \mathrm{Y}$ decay, and an analysis of the resulting dataset in the $1836 \mathrm{keV}$ line of ${ }^{88} \mathrm{Y}$ was used to confirm that this had been done correctly.

Figure 9 shows the results of spiskymax reconstruction of the extended source from dithered observations for different 


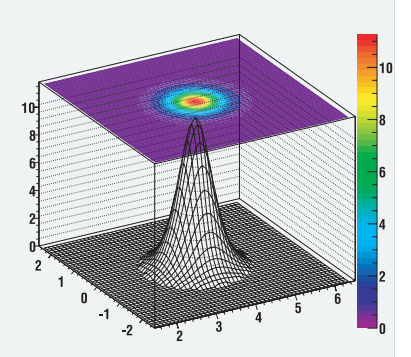

Fig. 6. Predicted emission from the extended $511 \mathrm{keV}$ source. (Diameter: $2.4^{\circ}$ )

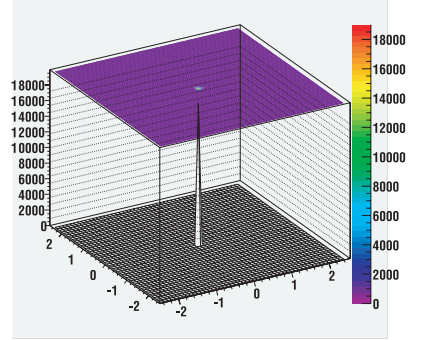

Fig. 7. spiskymax reconstruction of a ${ }^{22} \mathrm{Na}$ point source in the $511 \mathrm{keV}$ line from a non-dithered observation. Total $511 \mathrm{keV}$ flux is equal to that from the extended source in Fig. 8.

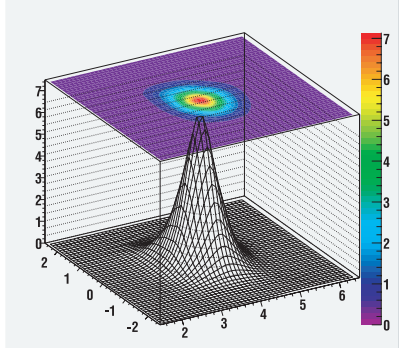

10 iterations

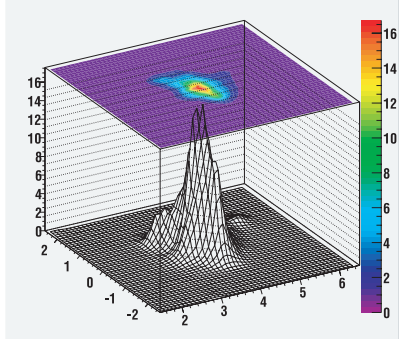

20 iterations

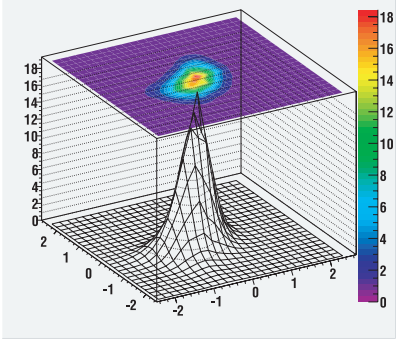

Fig. 8. spiskymax reconstruction of the $511 \mathrm{keV}$ extended source from a non-dithered observation. Total $511 \mathrm{keV}$ flux is equal to that from the point source in Fig. 7.

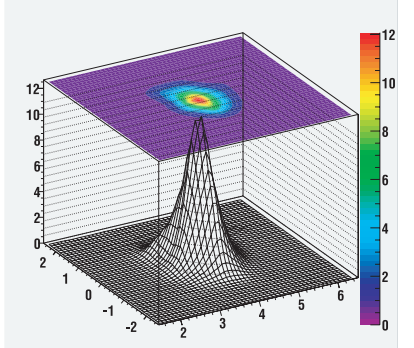

15 iterations

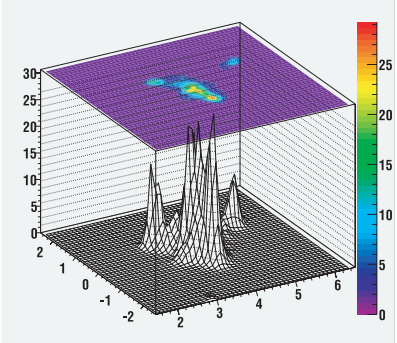

25 iterations

Fig. 9. spiskymax reconstruction of the $511 \mathrm{keV}$ extended source from dithered observations. Different iteration stages are shown.

numbers of iterations. The source is clearly visible in the images after 10 and 15 iterations. Its intensity, however, is still very much lower in the 10-iteration image than in the 15iteration image. Here, the flux begins to reach "final" reconstructed levels. Starting at iteration 20 - and very much so at iteration 25 and higher - the spiskymax-reconstructed image acquires more structure ("spikyness") than the original source distribution would merit. A (non-normalized) $\chi^{2}$ comparison

of calculated and reconstructed emission shows that the 15 and 20 iteration images best reflect the "true" distribution.

A similar behavior was observed when a simulated dithered observation of such an extended source was deconvolved with spiskymax. While for the determination of overall flux levels the number of iterations used is not too crucial, the reconstructed shape of the distribution depends on it. For the SPITS low background case, we found that the evolution of the reconstructed source flux and its significance help to determine a stopping criterion for spiskymax (Wunderer 2003).

The $511 \mathrm{keV}$ flux predicted from first interactions of $1.8 \mathrm{MeV}$ photons only in the $\mathrm{Pb}$ disk is $6.5 \times 10^{-3} \mathrm{ph} /\left(\mathrm{cm}^{2} \mathrm{~s}\right)$ while the reconstructed flux is $\sim 50 \%$ higher. In contrast, point source fluxes (from laboratory sources below $2 \mathrm{MeV}$ ) have always been reconstructed a few percent below the true flux. The deficiency in calculated flux from the extended source is likely due to a combination of (1) only the first interaction of a $1.8 \mathrm{MeV}$ photon being considered relevant in the calculation, while a once Compton-scattered photon with remaining energy of, say, $1.6 \mathrm{MeV}$ can still pair-produce in the $\mathrm{Pb}$ (add $\sim 6 \%$ ); (2) so far, the $2.7 \mathrm{MeV}$ line from ${ }^{88} \mathrm{Y}$ (emitted with $0.7 \%$ probability) has been neglected. Its contribution becomes non-negligible in this context due to the higher pair production cross section (add 5\%); (3) pair production interactions of source photons in the concrete wall behind the lead; and (4) $0.2 \%$ of ${ }^{88} \mathrm{Y}$ decays are $\beta^{+}$decays resulting in $511 \mathrm{keV}$ photons (this contributes another $\sim 11 \%$ to the observeable $511 \mathrm{keV}$ flux). Together, these effects enhance the $511 \mathrm{keV}$ flux from the region of the lead disk by roughly $20 \%$ - not accounting for pair production in the surrounding material which is not easily quantified -, enough to make the observed $(0.010 \pm 0.001) \mathrm{ph} /\left(\mathrm{cm}^{2} \mathrm{~s}\right)$ seem reasonable within the error margin.

\section{Conclusions}

With the SPI Imaging Test Setup, a single high-energy point source measured at high signal-to-background ratios can be localized to $0.1^{\circ}$ or better. Two such high-energy sources are reliably distinguished and localized by the spiskymax algorithm if they are separated by $1^{\circ}$ or more. Using spiskymax and dithering, a $2.4^{\circ}$-diameter extended $511 \mathrm{keV}$ source could be correctly imaged and its flux was reconstructed correctly within error margins. These SPITS results complement the lower-energy point source imaging tests performed with SPI during ground calibrations.

Acknowledgements. We gratefully acknowledge the help received at the IfS in Stuttgart from both the IfS team and people from MPE, especially A. Zoglauer. In addition, we wish to thank G. Vedrenne (CESR Toulouse) and F. Sanchez (U. Valencia) for making hardware available to SPITS.

\section{References}

Attié, D., Cordier, B., Gros, M., et al. 2003, A\&A, 411, L71

Badiali, M., Cardini, D., Emanuele, A., et al. 1985, A\&A, 151, 259

Bouchet, L., Jourdain, E., Roques, J.-P., et al. 2003, A\&A, 411, L377 
Connell, P., \& Skinner, G. K. 1995, in Proc. Imaging in High Energy Astronomy, ed. L. Bassani, \& G. di Cocco, 143

Connell, P. 1998, in Proc. of the 3rd INTEGRAL Workshop, ed. A. Bazzano, G. G. C. Palumbo, \& C. Winkler, 2, 397

Roques, J.-P., Schanne, S., v. Kienlin, A., et al. 2003, A\&A, 411, L91

Schanne, S., Cordier, B., Gros, M., et al. 2003, in SPIE 4851, 1132

Skinner, G. K., Connell, P. H., Naya, J., et al. 1997, in AIP Conf. Proc. 410, Proc. of the 4th COMPTON Symposium, ed. C. D. Dermer, M. S. Struckman, \& J. D. Kurfess, 1544

Skinner, G. K. \& Connell, P. 2003, A\&A, 411, L123

Strong, A. W., Diehl, R., Connell, P., \& Skinner, G. 1998, in Proc. of the 3rd INTEGRAL Workshop, ed. A. Bazzano, G. G. C. Palumbo, \& C. Winkler, 2, 221
Strong, A. W. 2003, A\&A, 411, L127

Sturner, S. J., Shrader, C. R., Weidenspointner, G., et al. 2003, A\&A, 411, L81

Vedrenne, G., Roques, J.-P., Schönfelder, V., et al. 2003, A\&A, 411, L63

Wunderer, C. B., Connell, P. H., Diehl, R., et al. 2001, IEEE Trans. Nucl. Sci, 48, 1053

Wunderer, C. B. 2003, Ph.D. Thesis, TU Munich, Germany, available on the www at http://tumb1.biblio.tu-muenchen.de/publ/diss/ph/ 2003/wunderer.html 